\title{
EFFICACY OF TRANSCUTANEOUS ELECTRICAL NERVE STIMULATION (TENS) IN THE MANAGEMENT OF CHRONIC NON BACTERIA PROSTATITIS PAIN: CASE STUDIES
}

\author{
Journal website at; \\ http://mrtbjournal.org/index.php/njmr/issue/current/showToc \\ *LAMINA S AND **HANIFS \\ *Department of Physiotherapy, Faculty of Medical Sciences, \\ Jimma University, Jimma, Ethiopia. \\ **Department of Physiotherapy, Faculty of Medicine, \\ Bayero University, Kano, Nigeria. \\ Correspondence to: \\ Lamina Sikiru \\ siklam_86@yahoo.co.uk
}

\begin{abstract}
SUMMARY
Objective: The aim of the study was to investigate the therapeutic efficacy of TENS in the symptomatic management of pain as a result of chronic prostatitis/ chronic pelvic pain syndrome.
\end{abstract}

Method: Two patients (A \& B) diagnosed with chronic prostatitis- category IIIA and IIIB of the National Institute of Health Chronic Pain (NIH-CP) referred for physiotherapy from the Urology department were recruited for this study. The third patient $(C)$ also diagnosed with chronic prostatitis category III A was recruited for the study from the Urology Department.

A pretest and posttest randomized design was used in data collection. Pre and post treatment pain level was assessed using the NIH-CP (pain domain) index. Patients $A$ and $B$ received TENS treatment, 5 times per week for a period of 4 weeks. The third patient $C$ was placed on antibiotics and analgesics.

Result: Findings of the study revealed the beneficial effect of TENS on chronic prostatitis pain in both patients A \& B.

Conclusion. Findings from the study concluded that TENS therapy could be an adjunct non invasive modality in the management of chronic pain as a result of chronic prostatitis /chronic pelvic pain syndrome.

Keywords: Pain; TENS; Chronic prostatitis; Bacteria.

\section{INTRODUCTION}

The National Institute of Health (NIH) classified prostatitis syndromes into four categories: ( I) Acute bacterial prostatitis; (II) Chronic bacterial prostatitis; (III)Chronic non bacteria prostatitis/chronic pelvic pain syndrome (CP/CPPS) (a) Inflammatory and (b)Noninflammatory; (IV)Asymptomatic inflammatory prostatiti (Krieger et al.,1999). This consensus classification recognizes pain as the primary component of the syndrome(Collins et al, 1998). Patients with category III(b) CP/CPPS have discomfort or pain in the pelvic region for at least 3 months with variable voiding and sexual symptoms in the absence of demonstrable infection. Patients with the inflammatory subtype of III (a)CP/CPPS have leukocytes in their expressed prostatic secretions; urine or semen. In contrast, those with the noninflammatory subtype (III b) have no evidence of inflammation (Gurunadha Rao Tunugutla \& Evans, 2002).

The etiology for chronic prostatitis/chronic pelvic pain syndrome (CP/CPPS) has not been fully elucidated and the current treatment strategies are not universally accepted (Krieger et al.,2003). Chronic infection, inflammation, neuropathy, pelvic floor muscle dysfunction, autoimmune disease and neurobehavioral disorders are some of the postulated etiologies though no single factor is thought to be the absolute cause. Standard therapies for CP/CPPS include antibiotics, anti-inflammatory agents, 5- $\alpha$ reductase inhibitors, and á-1 blockers (Krieger et al., 2003). In addition, it is not clear whether treatment for IIIa and IIIb prostatitis syndromes should differ because the role of inflammation in these pathological conditions is incompletely understood. (Gurunadha Rao Tunugutla \& Evans, 2002).

Recently, a multi-modal treatment approach and the utilization of complementary and alternative medicine 
(CAM) strategies such as acupuncture, phytotherapy, biofeedback, thermotherapy and physiotherapy have also been suggested as potential treatment options for CP/ CPPS (Shoskes, 2002; Chen \& Nickel, 2003).

Transcutaneous electrical nerve stimulation (TENS) was introduced as an alternative therapy to pharmacological treatments for chronic pain (Chao et al, 2007; Ying, 2007; Caroll et al, 2001; Akinbo et al,2000;). TENS currently is one of the most commonly used forms of electro analgesia in physiotherapy. Hundreds of clinical reports exist concerning the use of TENS for various types of conditions such as low back pain, myofascial and arthritic pain, sympathetically mediated pain, neurogenic pain, visceral pain, and post-surgical pain. Previous studies have established the beneficial effects of TENS in various chronic pain conditions (Ying \& While, 2007; Carroll et al., 2001; Akinbo, 2000). TENS is the application of pulsed square wave current through surface electrodes placed on the skin, to the peripheral nerve fibers for the control of pain. It is a non-invasive and non-addictive treatment and does not produce anaesthesia or nerve block (Akinbo,2003;Wall, 1985).

Studies have shown limited improvements in scores on the NIH Chronic Prostatitis Symptom Index with the use of biofeedback and acupuncture (Chen \& Nickel, 2003; Nedler, 2002). Physical therapies and sitz baths, have been recommended but have not been adequately studied (Nickel et al, 2007; Schaeffer, 2006). The needs for the symptomatic management of pain in CP/CPPS with a noninvasive, non-addictive technique such as TENS clearly exist. The purpose of the present study was therefore to determine the efficacy of TENS in the symptomatic management of pain as a result of chronic prostatitis/ chronic pelvic pain syndrome.

\section{Methodology}

Subject Selection

The participants for this study included 3 diagnosed CP/CPPS patients attending the Urology department of Murtala Mohammad Specialist Hospital (MMSH) Kano and from private urologists.

\section{Inclusion and Exclusion Criteria}

The inclusion criteria were men between $30-50$ years, previously diagonized as category IIIA or IIIB CP/CPPS

\section{Exclusion Criteria}

Exclusion criteria were prostate and other urinogenital cancer and infection, loss of skin sensation at and around painful area, cardiac pace maker, previous exposure to TENS and other electro analgesia.

\section{CASE REPORTS}

Ethical approval and consent:

The 3 patients for the study gave their consent prior to the commencement of the study and Ethical approval was obtained from the Ethical Committee of the Murtala
Mohammed Specialist Hospital, Kano, Nigeria.

\section{Patients' Clinical History:}

Patient A: A 50-year old civil servant reported in the urology department of Murtala Mohammad Specialist Hospital (MMSH), Kano; where he was diagnosed as a case of CP/CPPS of 15 months history. Patient has been on medication (series of antibiotics and analgesics) for a very long time ( $>6$ months), all to no avail. Patient complained of shooting supra-pubic pain radiating to the penis. Patient was off analgesics due to the development of gastritis and was placed on only antibiotics and referred for physiotherapy.

Patient B: A 30 year old trader reported symptoms of pin and needle sensation, also painful supra-pubic and lower abdominal since the past 1 year. Patient was diagnosed as $\mathrm{CP} / \mathrm{CPPS}$ and was placed on medication (antibiotics and analgesics) and was referred for physiotherapy.

Patient C: A 32 year old artisan was from a private clinic to the urology unit of MMSH where he was diagnosed as CP/CPPS of 2 year's history. Patient complained of chronic lower abdominal pain, perinea pain and discomfort. Patient was placed on antibiotics and analgesics by the Urologist.

\section{EXAMINATIONANDEVALUATION}

Patient A: The NIH chronic prostatitis symptom index questionnaire (NIH-CPSIQ), the pain domain describing the location, frequency and severity of pain was presented the patient and instruction was given to indicate the pain characteristics and level by signifying a number on the scale (Litwin et al., 1999). Patient indicated a sum of 19

Patient B: Patient indicated 20 on the NIH-CPSIQ, the pain domain (see appendix A).

Patient C: Patient indicated 19 on the NIH-CPSIQ pain domain.

\section{TREATMENT}

\section{Pre-treatment procedure}

Patients A \& B (antibiotics + TENS): Both patients A and $B$ were screened for all the contra-indications to TENS (numbness, broken skin, skin infection/irritation etc). Both patients continued their antibiotics ofloxacin (300mg Trice daily) as prescribed. Sensory test was conducted on the skin over the painful area by using two test tubes with cold and warm water, also light touch (using soft brush). It was ascertained that sensitivity of the area was intact, and that there was no resistance, this allowed for effective stimulation.

Treatment procedure: For TENS application, patients A \& B were comfortably positioned supine lying based on the painful area to expose the perineal-suprapubic region for electrode placement. TENS gel was applied on the surface of the electrodes to aid maximum transmission of current. Electrodes were placed on the skin overlying the 
painful area and held firmly in position (Oosterhof et al., 2006; Radhakrishnan \& Sluka, 2005).

The machine was switched on; a suitable and comfortable frequency and pulse width were selected on the stimulator by turning the appropriate knobs. Intensity knob was turned to a level when the patients felt a tingling or pins and needle sensation, the intensity was then reduced to a level that the patient reported a comfortable stimulation.

Patients were stimulated with high TENS (Acupuncture like TENS) daily for an average of 20 minutes, mean frequency, pulse width and intensity of $100 \mathrm{~Hz}, 100 \mu \mathrm{s}$ and $25 \mathrm{~mA}$ respectively for a mean treatment parameters of 3 times per week for 4 consecutive weeks (average of 12 treatment sessions).

Seven days prior to their next medical consultation, after which both patients (A \& B) had exhausted their antibiotics and TENS treatment had stopped (7 days post treatment [wash out period]), both patients were assessed for the Post-treatment pain score using the same pre treatment procedure.

Patient $\mathbf{C}$ (antibotics + analgesic): Patient $\mathbf{C}$ continued with antibiotics (ofloxacin) and analgesics (ibuprofen $400 \mathrm{mg} \mathrm{b.d)}$ as prescribed by the urologist for the same period of time ( 4 weeks). This patient received no TENS management. Also, seven days prior to their next medical consultation, after which this patients $(C)$ had exhausted the prescribed analgesic and antibiotics ( 7 days post treatment [wash out period]), patient $C$ was also assessed for the Post-treatment pain score using the same pre treatment procedure.

Post-treatment procedure: At the end of the total treatment period and 7 days post TENS and NSAIDs treatment(wash out period), The NIH-CPSIQ (pain domain) was presented to the 3 patients to indicate their post treatment pain level. Patient A reported 9, and patient B reported 11 on the NIH-CPSIQ, while patient C reported 16 (table 1 ).

Table 1: Subjects treatment responses pre and post treatment

\begin{tabular}{llll}
\hline Subject & $\begin{array}{l}\text { Pre-treatment } \\
\text { Scores } \\
\text { NIH-CPSIQ } \\
\text { Scores }\end{array}$ & $\begin{array}{l}\text { Post-treatment } \\
\text { Scores } \\
\text { NIH-CPSIQ } \\
\text { Scores }\end{array}$ & $\begin{array}{l}\text { \% pain } \\
\text { reduction }\end{array}$ \\
\hline Patient A & 19 & 9 & 53 \\
Patient B & 20 & 11 & 45 \\
Patient C & 19 & 16 & 15 \\
\hline
\end{tabular}

\section{DISCUSSION}

The purpose of this case reports is to document the therapeutic efficacy of TENS in the symptomatic management of chronic pain in CP/CPPS. The result showed an appreciable effect of TENS in the symptomatic management of chronic pain in CP/CPPS. The predominant symptom of CP/CPPS is pain. Therefore, modalities to treat pain specifically may be effective. There is mounting evidence that the pain of CP/CPPS may be neuropathic and associated with central nervous system changes(Chen \& Nickel, 2003). The presence of central sensitization in patients with CP/CPPS was demonstrated by Yang and colleagues (Yang et al., 2005), who compared thermal algometry in men with CP/CPPS versus asymptomatic controls. Men with CP/CPPS reported a higher visual analogue scale to short bursts of noxious heat stimuli to the perineum but no difference to the anterior thigh. Thus, these patients have altered sensation in the perineum compared with controls.

Many studies have investigated the effects of complementary and alternative medicine (CAM) strategies in the management of CP/CPPS. The result of the present study was in agreement with a similar non invasive CAM therapy, reported by Capidice et al, (2007) .In their pilot study; they investigated the effect of acupuncture in 10 men diagnosed as CP/CPPS (category IIIA or IIIB).Acupuncture was applied for 30 minutes, twice weekly for 6 weeks. They reported significant decrease in NIH-CPSI for pain and lower urinary tract symptoms and quality of life.

Another study with a similar result with present study was conducted by John and co workers in 2003. Their study tested a high frequency, urethral-anal prototype stimulation device in men with CP/CPPS twice weekly for 5 weeks. The results demonstrated a significant decrease in the NIH-CPSI $(P=0.0002)$ with no urethral, anal complaints or other side effects The authors suggest that due to the positive results, simple technology and ability to be self-administered, this new device may have utility in the treatment of CP/CPPS.

Two similar studies on non pharmacological, non invasive CAM therapy testing the value of biofeedback therapy for CP/CPPS yielded positive results (Ye et al, 2003; Clemens et al, 2000). The first study (Yee et al., 2003) assessed 62 patients who were refractory to conventional therapy (such as antibiotics and/or alpha-blockers) for greater than half a year. These patients were treated utilizing the Urostym Biofeedback equipment five times a week for 2 weeks with a stimulus intensity of 15-23 mA and duration of $20 \mathrm{~min}$. The NIH-C.PSI index noted a significant overall reduction in score $(P<0.01)$ and no side effects were reported during the trial.

The second pilot study (Clemens et al., 2000) evaluated biofeedback therapy in 19 men with pelvic floor tension and CP/CPPS. These results demonstrated significant improvement in pain scores as measured by the American Urological Association (AUA) symptom index $(P=0.001)$. While this study focused on testing the effect of biofeedback therapy in treating the symptoms associated with CP/CPPS, it also implicated the presence of pelvic floor tension contributing to pain and the paramount importance of muscular reeducation for its treatment (Clemens et al., 2000). These initial, positive biofeedback studies may warrant larger randomized clinical 
trials to confirm safety and efficacy as well as explore the mechanism of action of biofeedback therapy.

Previous studies have reported significant effect of TENS on visceral pain such as labour pain and dysmenorrhoea (Chao et al., 2007; Ying \& While, 2007; Akinbo et al, 2000). These previous findings are in line with the report of the present finding on similar visceral organ. There is no better way of eliminating pain than by removing its cause, with any symptomatic therapy, however, efficacy must be weighed with the risks involved. TENS might be preferable to large amount of analgesics and their side effects. Also, TENS is readily available to both patients and therapists, cheaper and easy to apply compared to other non invasive, non pharmacological CAM therapies such as acupuncture, biofeedback, heat therapy or hyperthermia(transrectal microwave therapy)

\section{CONCLUSION}

This report had demonstrated the therapeutic efficacy of TENS in the symptomatic management of CP/ CPPS. While our findings provide a rational base for recommending TENS in the symptomatic management of CP/CPPS pain, further studies using larger samples are needed to evaluate the therapeutic efficacy of TENS in the management of CP/CPPS . Also future studies should further evaluate the effect of TENS on other domains (urinary symptom \& quality of life impact domains) and total NIH-CPSI scores.

\section{REFERENCES}

- Akinbo SRA, Onwudimegwu WN, Ajayi GO (2000) Evaluation of the efficacy of TENS compared with analgesics in the management of primary dysmenorrhoea. Journal of Nigeria Medical Rehabilitation Therapists. 5(10): 27-30.

- Akinbo SRA, Oyedele SY, Shaba OP (2003). Transcutaneous electrical nerve stimulation (TENS) in the management of temporomandibular joint pain and dysfunction syndrome. Journal of the Nigeria Medical Rehabilitation Therapists. 8(2): 32-35.

- Capodice JL, Jin Z, Benis DL, Samadi D, Stone BA, Kapan S, Katz AE .(2007) A'pilot study on acupuncture for lower urinary tract symptoms related to chronic prostatitis/chronic pelvic pain syndrome. Chin Med. 2: 1.dio:10:1186/17478546- b2- 1

- Carroll D, Moore RA, McQuay HJ, Fairman F, Tramen M, Leijon G. (2001). Transcutanous electrical nerve stimulation (TENS) for chronic pain. Cochrane Database Syst. Review. 3: CD003222

- Chao AS, Chao A, Wang TH, Chang YC, Peng HH, Chang SD, Chao A, Chang CJ, Lai CH, Wong AM.(2007) Pain relief by applying TENS on acupuncture points during the first stage of labour: a randomized double blind placebo controlled trial. Pain. 127: $214-20$.

- Chen R, Nickel JC. (2003) Acupuncture ameliorates symptoms in men with chronic prostatitis/chronic pelvic pain syndrome. Urology. 61: 1156-1159.
- Clemens JQ, Nadler RB, Schaeffer AJ, Belani J, Albaugh J, Bushman W.(2000) Biofeedback, pelvic floor re-education, and bladder training for male chronic pelvic pain syndrome. Urology. 56: 951-5

- Collins MM, Stafford RS, O'Leary MP, Barry MJ. (1998) How common is prostatitis? A national survey of physician visits. J Urol. 159: 1224-8.

- Gurunadha Rao Tunuguntla HS, Evan C P. (2002) Management of prostatitis. Prostate Cancer and Prostatic Diseases 5(3): 172-179.

- John H, Ruedi C, Kotting S, Schmid DM, Fatzer M, Hauri D. (2003) A new high frequency electrostimulation device to treat chronic prostatitis. $J$ Urol. 170: 1275-7.

- Kreiger JN, Ross SO, Penson DF, Riley DE.(2002): Symptoms and inflammation in chronic prostatitis/chronic pelvic pain syndrome. Urology. 60: 959-963.

- Krieger JN, Nyberg L Jr, Nickel JC.(1999) NIH consensus definition and classification of prostatitis. JAMA. 282: 2367.

- Litwin MS, McNaughton-Collins M, Flowler FJ.(1999) The NIH Chronic Prostatitis Index [NIH-CPSI]:Development and validation of a new outcome measure. J Urol. 162: 369375.

- Nadler RB.(2002) Bladder training biofeedback and pelvic floor myalgia. Urology. 60 (Suppl 6): 42-43.

- Nickel JC, Baranowski AP, Pontari M, Berger RE, Tripp DA. (2007) Management of Men Diagnosed With Chronic Prostatitis/Chronic Pelvic Pain Syndrome Who Have Failed Traditional Management. Rev Urol. 9(2): 63-72.

- Oosterhof J, De Boo TM, Oostendorp RA, Wilder-Smith OH,Crul BJ (2006) Outcome of TENS in chronic pain : short term results of a double blind randomized, placebo controlled trial. J Headache Pain. 7(4): 196-205.

- Radhakrishnan R, Sluka KA.(2005) Deep tissue afferent , but not cutanous afferents mediate TENS induced antihyperalgesia. J Pain. 69(10): 673-80.

- Schaeffer AJ. (2006) Chronic Prostatitis and the Chronic Pelvic Pain Syndrome.Clinical Practice. 355(16): 1690-1698

- Shoskes DA.(2002) Phytotherapy and other alternative forms of care for the patient with prostatitis. Curr Urol Rep. 3: 330-4.

- Wall PD. (1985) The discovery of TENS. Physiotherapy. 71: $348-350$.

- Yang CC, Lee JC, Kromm BG.(2003) Pain sensitization in male chronic pelvic pain syndrome: why are symptoms so difficult to treat? J Urol. 170: 823-827

- Ye ZQ, Cai D, Lan RZ, Du GH, Yuan XY, Chen Z.(2003) Biofeedback therapy for chronic pelvic pain syndrome. Asian J Androl. 5:155-8.

- Ying KN, While A.(2007) Pain relief in osteoarthritis and rheumatoid arthritis : TENS. Br J Community Nurs. 12(8): $364-71$. 